\title{
Surgical Treatment of Cavernous Sinus Cavernomas: Evidence from Vietnam
}

\author{
Duc-Anh Nguyen ${ }^{1}$, Hao The Nguyen ${ }^{1}$, Thang Van Duong ${ }^{1}$, Binh Hoa Pham ${ }^{2}$ and \\ Hoang-Long Vo ${ }^{3, * \mathbb{D}}$ \\ 1 Department of Neurosurgery, Bach Mai Hospital, Hanoi 100000, Vietnam; \\ anhducyhnhmu@gmail.com (D.-A.N.); ngthehao2002@gmail.com (H.T.N.); \\ duongvanthangbmhs@gmail.com (T.V.D.) \\ 2 Department of Neurosurgery, 108 Military Central Hospital, Hanoi 100000, Vietnam; \\ phbinh108.hpt@gmail.com \\ 3 Institute for Preventive Medicine and Public Health, Hanoi Medical University, Hanoi 100000, Vietnam \\ * Correspondence: vohoanglonghmu@gmail.com; Tel.: +84-98-540-6430
}

Received: 1 March 2020; Accepted: 13 May 2020; Published: 23 May 2020

\begin{abstract}
Cavernous sinus cavernomas, a rare vascular malformation, represents $3 \%$ of all benign cavernous sinus tumors. Both clinical and radiological signs are important for differentiating this condition from other cavernous sinus diseases. The best treatment is radical removal tumor surgery; however, due to the tumor being located in the cavernous sinus, there are many difficulties in the surgery. We report a case of a 35-year-old female who only presented sporadical headache. After serial magnetic resonance imaging acquisitions, a tumor measuring $30 \mathrm{~mm}$ in the left cavernous sinus and heterogenous enhencement was observed. Then, the patient underwent an operation with an extradural basal temporal approach. Postoperatively, the tumor was safely gross total removed. The patient developed left oculomotor nerve palsy but fully recovered after 3 months of acupunture treatment, and developed persistent left maxillofacial paresthesia. The surgical treatment for cavernous sinus cavernomas may be considered a best choice regarding safety and efficiency.
\end{abstract}

Keywords: cavernous sinus; cavernoma; tumors; meningioma; oculomotor nerve palsy

\section{Introduction}

Cavernomas, a fourth common cerebral vascular malformation, can be diagnosed easily by magnetic resonance imaging [1]. However, cavernomas in the cavernous sinus is very rare and may be misdiagnosed with several other tumors, such as meningiomas, schwannomas, or pituitary adenomas. Cavernomas is a type of benign vascular tumor, and the patients with cavernomas can be treated if the tumor is totally removed. However, the tumor approach and resection is very difficult with a high risk of morbidity and mortality due to the cavernomas being located in the cavernous sinus. Herein, we present a case of cavernous sinus cavernomas (CSC) that was successfully operated on at a central hospital and review the literature.

\section{Case Presentation}

A 34-year-old female presented with a history of occassional headache without remarkable physical and neurological symptoms. Routine laboratory investigations and pituitary hormonal tests were all normal. She was taken for a magnetic resonance imaging and a mass was discovered in the region of the sellar and left parasellar. The size of the lesion was around $30 \mathrm{~mm}$. It elicited a high signal on T1-weighted and T2-weighted images, strong enhanced gadolinium, and a homogenous intense 
surrounding left cavernous internal carotid, pushing downward on the skull base and pushing the pituitary contralaterally (Figure 1).

Then, the patient underwent surgery to have resection via the extradural subtemporal approach. After craniotomy and exposing the way to the cavernous lateral wall, the rotundum foramen and ovale foramen were continuously dissected. The dural layer of the cavernous lateral wall was rolled up to expose the second and third branches of the trigeminal nerve and the Gasser ganglion. The maxillary nerve was sacrificed by using a sharp knife to open the internal layer of the cavernous sinus along the route of the nerve to access the tumor. At this point, the tumor was very soft, friable, and compressible by cotton or spongel though it easily bled. The lesion was debulked, and controlled suction was performed to remove the bulk to nearly total tumor removal after exposing the cavernous segment of the internal carotid artery.

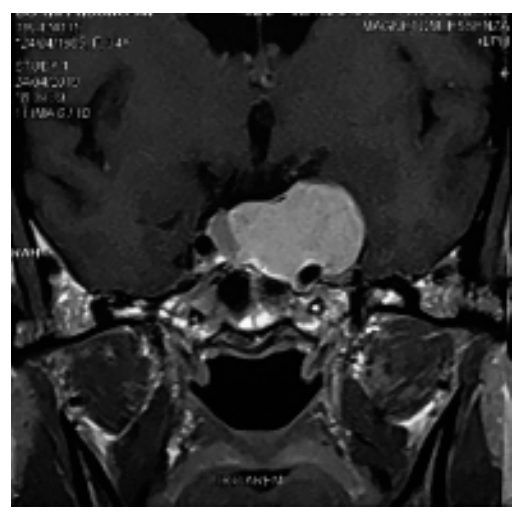

(a)

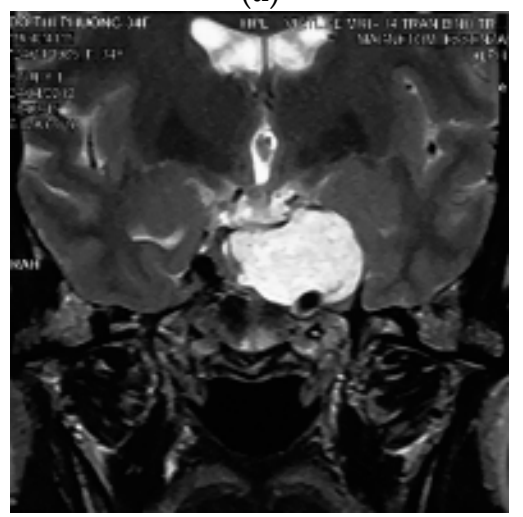

(c)

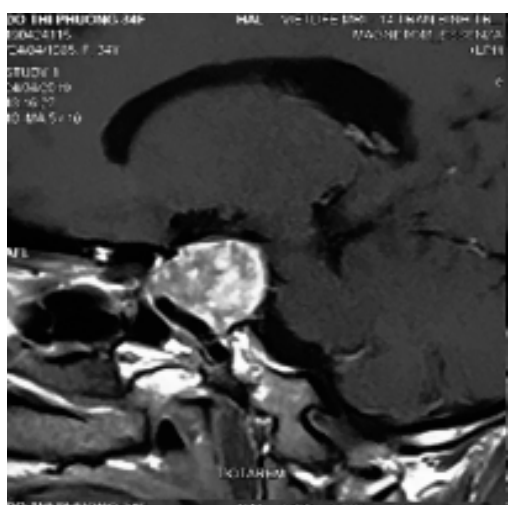

(b)

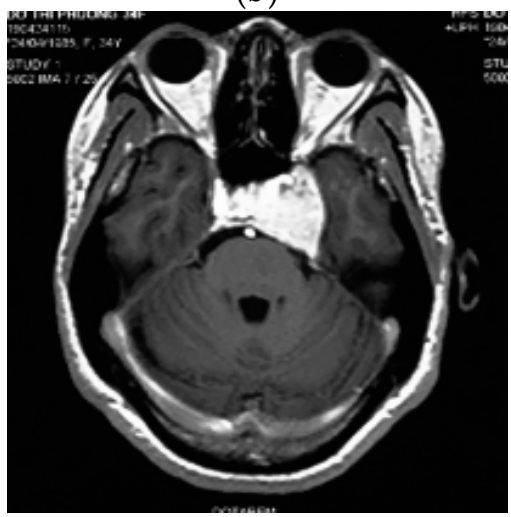

(d)

Figure 1. Contrast-enhanced magnetic resonance imaging examination of the brain. $(\mathbf{a}, \mathbf{b})$ Pre contrast coronal and sagital T1w showed an extra axial sellar and left parasellar mass with hyperintensity, the cavernous internal carotid artery is pushed inferiorly and encased by the lesion. (c) Coronal T2w showed a high signal intensity of the mass. (d) Initial gadolinium-enhanced axial T1w showed heterogenous enhancement of the mass.

Histopathological examination of the mass revealed a cavernous haemangioma with many large cystically vessels filled by blood cells with an irregular thickness of the walls covered by benign flat endothelial cells (Figure 2). After surgery, the patient presented left ptosis, left eye exotropia (due to trauma of the third nerve), and left maxillary numbness (due to trauma of the V2 nerve) (Figure 3). However, the symptoms of third nerve palsy completely recovered after rehabilitation for 3 months. At the moment, the patient only has permanant numbness in the left cheek.

All procedures performed in study involving human participant was in accordance with the ethical standards of the institutional and/or national research committee and with the 1964 Helsinki declaration and its later amendments or comparable ethical standards. The patient and her family were 
informed about our intension to involve her in a case study and they agreed to partake in the study. They signed the concern form before the operation was carried out according to all surgical protocols.

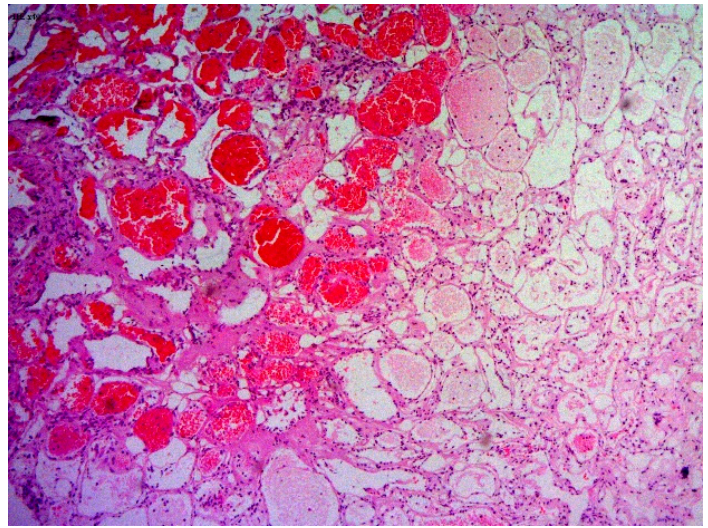

(a)

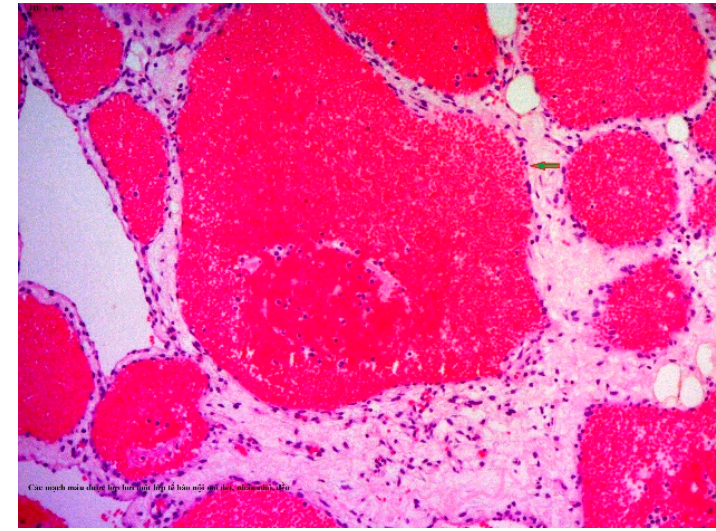

(b)

Figure 2. Histopathology revealing large numbers of thin-walled vascular sinusoids, with a single layer of endothelium-lined capillaries, and scanty connective tissue. (a) H\&E, 100×; (b) H\&E, 250×. $\mathrm{H} \& \mathrm{E}$, hematoxylin and eosin.

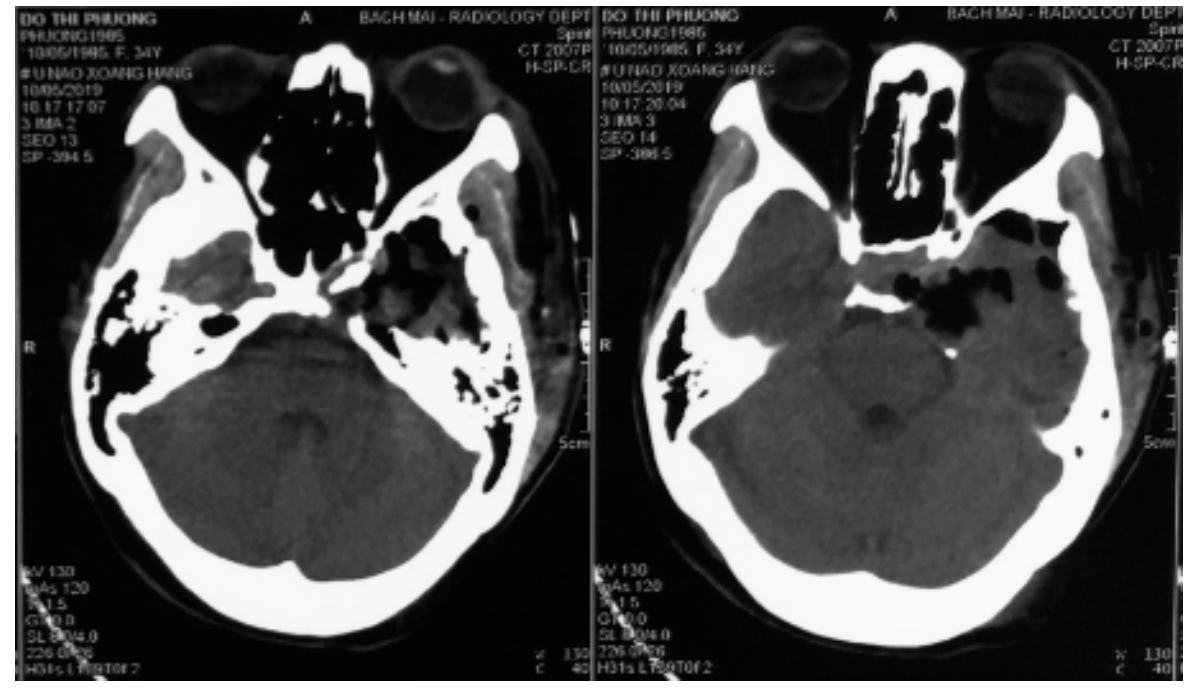

Figure 3. Two-day postoperative computed tomography scan images.

\section{Discussion}

Cavernomas haemangiomas, also known as cavernous angiomas or cavernomas, are benign tumors defined as vascular malformations formed of abnormal dilated vascular spaces with no intervening neural tissue [2]. Most of them occur within cerebral parenchyma,; however, they are sometimes found in the lateral wall of the ventricle, brainstem, cerebellum, basal ganglions, dura, and pituitary fossa [3]. Lesions located in the cavernous sinus are very rare [1]. At this region, it is neccessary to differentiate from some other lesions, such as meningiomas, schwannomas, pituitary macroadenomas, lymphomas, metastase tumor, dermatomas, or aspergillomas. In terms of epidemiology, CSCs usually present in middle-aged females, about 50 years old [4-6]. Regarding histopathology, CSCs have characteristics that are similar to lesions of the intracerebral parenchyma but have a different clinical picture and radiologic findings [7].

Initial symptoms are usually headache and dysfuntion of the cranial nerves passing through the cavernous sinus, presenting as ptosis, diplopia, or facial neuralgia, and decreased visual acuity. These are non-specific because many lesions in the cavernous sinus can cause symptoms like this. 
In the magnetic resonance imagings, the lesion revealed an enhanced homogenous mass encircling the cavernous segment of the internal carotid artery without occluding it. This might be related to the natural character of the tumor. With cavernomas in the cavernous sinus, the blood stream through the lesion was homogenous, contrary to cavernomas intracerebral parenchyma, with no bleeding inside the tumor, and no calcification. Cerebral cavernomas appeared as "popcorn-shaped" or "berry-shaped", which showed isointense in magnetic resonance imagings with a rim of signal loss due to hemosiderin. This was a specific radiological feature of parenchymal cavernomas, but it seldom happens with extra axial cavernomas, especially in the cavernous sinus [7]. In the magnetic resonance imaging, CSCs were homogenous; hyperintense in T1w, T2w, and T2 Flair; showed contrast enhancement; and rarely had intratumor bleeding or calcification (Figure 1).

A computed tomography scanner and magnetic resonance imaging can be used to differentiate from three other common lesions, which are meningiomas, schwannomas, and pituitary microadenomas [8]. Meanwhile, CSCs present as a well-demarcated mass, isointense or hypointense on T1w compared to the surrounding cerebral tissue, meningiomas are much more isointense, and schwannomas have a tendency to be hypointense. All of them have contrast enhencement.

On the radiological images, CSC appears with a clear boundary, and isointense or slightly hyperintense in $\mathrm{T} 1 \mathrm{w}$ and $\mathrm{T} 2 \mathrm{w}$ compared to parenchymal tissue. Whereas, meningiomas are more isointense, while schwannomas tend to have a slightly low density compared to brain parenchyma. All three types of these tumors show contrast enhancement after the injection. However, schwannomas often catch contrast material irregularly and are speckled due to the alternation between the regions of increased and decreased signal, while meningiomas and CSCs can catch contrast material quite uniformly. In addition, the presence of calcification clumps in the tumor or the presence of bone erosion around the tumor is more likely to suggest meningeal lesions $[3,9]$. This phenomenon is less common in schwannomas and cavernous angiomas in the sinus cavity.

Most CSCs have sinus occlusion images, or even complete obstruction on the angiography film [1]. The angiographic images in patients with schwannomas tumors are variable, with no characteristic signs. As for meningiomas, the images reveal a strong vascularization and strong enhancement, homogeneously late after injection.

Schwannomas tends to be more hypointense than the brain parenchyma in the $\mathrm{T} 1 \mathrm{w}$, and homogeneously hyperintense in the $\mathrm{T} 2 \mathrm{w}$. Whereas, meningiomas usually have a similar signal, with the parenchymal tissue both in $\mathrm{T} 1 \mathrm{w}$ and $\mathrm{T} 2 \mathrm{w}$. Both types of tumors have strong enhancement and are quite homogeneous with meningiomas. For CSC, the lesions had a clear boundary with the surrounding structures, increasing the signal or a mixed signal in T1w and T2w. However, unlike the parenchymal cavernomas, the tumor was not hypointense at the edge [10]. After magnetic contrast injection, the tumor was very strong and showed relatively homogeneous enhancement. This feature was similar to previous reports of cavernous hemangiomas in the cavernous sinus cavity $[4,5,8]$. Occasionally, an increased signal image on T2-weighted images has also suggested lesions of the CSC. With other lesions in the cavernous sinus, such as metastatic tumor, granuloma, chordoma, lymphoma, and aspergilloma, this showed as hyperintense in $\mathrm{T} 2 \mathrm{w}$, but the signal was not homogeneous and the boundary was not clear.

Accurate diagnosis of the tumor is extremely important, because it involves surgical tactics and surgical procedures [8]. In essence, CSC is benign, slowly growing, and absolutely curable if the tumor is completely removed by surgery. However, operation is a huge challenge because of the tumor location in the cavernous sinus, high risk of bleeding, difficulty in stopping the bleeding, as well as the risk of damage to the cranial nerves located in the sinuses (such as III, IV, V, VI nerves) [11,12]. Previous reports showed that the rate of total removal of tumors by microsurgical surgery was only $30-44 \%$ [13], while the complication rate was very high. Therefore, resection of CSC is considered a dangerous procedure.

Various methods have been proposed, such as inserting a balloon into the carotid artery to reduce blood flow through the sinuses and reduce bleeding [11,14], pre-opt radiotherapy to reduce vascular growth $[11,15,16]$, injecting a vasoconstrictor into the tumor (such as alcohol, and bio fibrin glue) $[17,18]$, 
inducing hypotension during surgery [19], and using an autologous blood recovery machine during surgery [20].

In terms of the surgical approach, each of the authors had their own choice, but all of them discussed requests, such as the ability of expanding the surgical field, controlling the internal carotid artery, preserving cranial nerves, and limitting blood loss. Goel et al. chose the frontal subtemporal approach [21], while several other authors have chosen the frontotemporal orbital zygomatic approach (FTOZ approach) [22]. In this circumstance, we used the extradural subtemporal approach owing to the ability to control bleeding. In addition, intradural dissection risks temporal lobe contusion, causing post-operation seizure.

After detaching the dura from the skull base towards the cavernous sinus, the dura was continuously rolled up to reveal V3, V2, and V1. This was a very safe non-bleeding method and was clearly shown to be effective in Goel's study (1997) [23] and Li's study (2015) [4]. The V2 branch was chosen to sacrifice to make entry because this nerve only has a feeling function for the ipsilateral maxillary area and its position is safer, resulting in low risk to damage of the internal carotid artery and cranial nerves in the cavernous sinus cavity.

After incising the inner layer of the lateral wall of the cavernous sinus, along the path of the V2 branch nerve and partial resection this nerve, the tumor inside the sinus was exposed as a soft porous mass that was easy to bleed but it could be stopped by squeezing or spongel. The tumor could be completely dissected, and separated from the carotid artery and the sinus cavity with a blunt tool to avoid damaging the blood vessels in the sinus. Some authors suggested that CSC should be removed in bloc dissection [8,11,24]; however, this method is only suitable for small and medium tumors. For large tumors, they should be taken in small pieces (debulking dissection) to stop bleeding with pressed cotton, which will reduce blood loss and bleeding during surgery. In the places where veins flow into the cavernous sinus, after tumor resection, we used a spongel inserted into it to stop the bleeding. With the bleeding from the artery feeders, mini clips were used, and electronical coagulation was limitted due to ICA constriction. We observed that this method of hemostasis was very effective, which ensured that the surgical field was not flooded by blood, helping the dissection and resection process to be increasingly more safe and reducing blood loss. During the operation, the sinus cavity was opened in a straight line, parallel to the V2 nerve; hence, as the dissection on the arch of the sinus cavity, we had to lift the lateral wall of the cavernous sinus upward. This manipulation might have damaged the thrid nerve, leading to thrid nerve paralysis postoperatively. However, this condition was temporary, as the third nerve was almost fully recovered after acupuncture for 3 months. The phenomenon of maxillary facial numbness (corresponding to the V2 branch) has been predicted intra surgery. Postoperative magnetic resonance imaging showed that the tumor was mostly removed (Figure 4).

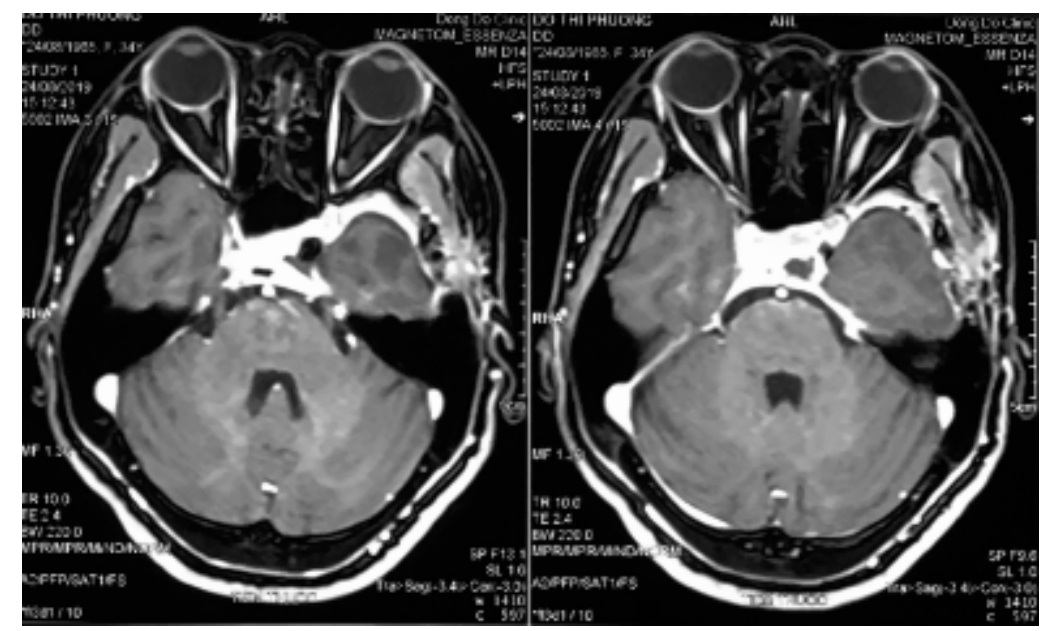

Figure 4. Cont. 


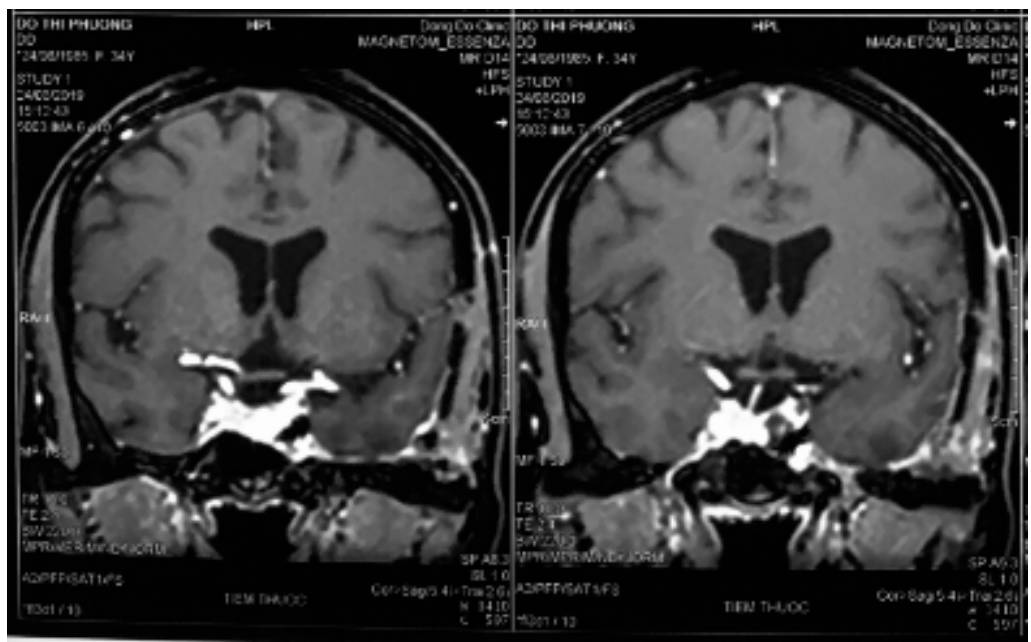

Figure 4. Three-month postoperative magnetic resonance imaging.

\section{Conclusions}

Cavernoma of the cavernous sinus is a rare vascular malformation with no specifically clinical signs, requiring a differential diagnosis from meningiomas, schwannomas of the cranial nerve, and pituitary adenomas in the sinus cavity. Tumor removal is really challenging due to the location in the sinus cavity, high vascularity, many cranial nerves, and the carotid artery surrounding. However, from the Vietnamese authors's experience of successful resection of a cavernoma of the cavernous sinus by the extradural subtemporal approach and stop-bleeding techniques, the ability of removing an entire tumor is positive, offering good treatment results for such cases.

Author Contributions: Conceptualization, D.-A.N. and H.T.N.; Methodology, D.-A.N., T.V.D., and B.H.P.; Investigation, D.-A.N. and H.T.N.; Data Curation, D.-A.N., H.T.N., B.H.P., T.V.D. and H.-L.V.; Writing-Original Draft Preparation, D.-A.N. and H.-L.V.; Writing-Review \& Editing, D.-A.N. and H.-L.V.; Visualization, T.V.D. and B.H.P.; Supervision, H.T.N., B.H.P. and T.V.D. All authors have read and agreed to the published version of the manuscript.

Funding: This research received no external funding.

Acknowledgments: All authors sincerely thank the patient and her families. All members working at Department of Neurosurgery (Bach Mai Hospital) during the treatment time are gratefully acknowledged.

Conflicts of Interest: The authors declare no conflict of interest.

\section{References}

1. Lupret, V.; Negovetić, L.; Smiljanić, D.; Klanfar, Z.; Talan-Hranilović, J. Cavernous haemangioma in cavernous sinus: Case report of rare location. Neurol. Croat. 1992, 41, 241-246.

2. Rosenblum, B.; Rothman, A.S.; Lanzieri, C.; Song, S. A cavernous sinus cavernous hemangioma: Case report. J. Neurosurg. 1986, 65, 716-718. [CrossRef] [PubMed]

3. Goto, Y.; Yamabe, K.; Aiko, Y.; Kuromatsu, C.; Fukui, M. Cavernous hemangioma in the cavernous sinus. Neurochirurgia 1993, 36, 93-95. [CrossRef] [PubMed]

4. Li, M.-H.; Zhao, J.L.; Li, Y.Y.; Zheng, C.H.; Xu, G.S.; Hong, T. Extradural transcavernous approach to cavernous sinus cavernous hemangiomas. Clin. Neurol. Neurosurg. 2015, 136, 110-115. [CrossRef]

5. Li, H.; Zhang, B.; Wang, W.; Wei, M.H.; Liu, B.Y.; Wu, Z. Clinical Features, Intradural Transcavernous Surgical Management, and Outcomes of Giant Cavernous Sinus Hemangiomas: A Single-Institution Experience. World Neurosurg. 2019, 125, e754-e763. [CrossRef]

6. Yin, Y.-H.; Yu, X.G.; Xu, B.N.; Zhou, D.B.; Bu, B.; Chen, X.L. Surgical management of large and giant cavernous sinus hemangiomas. J. Clin. Neurosci. 2013, 20, 28-133. [CrossRef]

7. Lombardi, D.; Giovanelli, M.; De Tribolet, N. Sellar and parasellar extra-axial cavernous hemangiomas. Acta Neurochir. 1994, 130, 47-54. [CrossRef] 
8. Ibrahim, D.; El Fiki, A.; Hafez, M.; Saleem, S. Report of a case of cavernous haemangioma of the cavernous sinus. BJR Case Rep. 2019, 5, 20190031. [CrossRef]

9. Lee, A.G.; Miller, N.R.; Brazis, P.W.; Benson, M.L. Cavernous sinus hemangioma. Clinical and neuroimaging features. J. Neuroophthalmol. 1995, 15, 225-229. [CrossRef]

10. Katayama, Y.; Tsubokawa, T.; Miyazaki, S.; Yoshida, K.; Himi, K. Magnetic resonance imaging of cavernous sinus cavernous hemangiomas. Neuroradiology 1991, 33, 118-122. [CrossRef]

11. Linskey, M.E.; Sekhar, L.N. Cavernous sinus hemangiomas: A series, a review, and an hypothesis. Neurosurgery 1992, 30, 101-1088. [CrossRef] [PubMed]

12. Namba, S. Extracerebral cavernous hemangioma of the middle cranial fossa. Surg. Neurol. 1983, 19, 379-388. [CrossRef]

13. Park, C.K.; Choi, S.K.; Kang, I.H.; Choi, M.K.; Park, B.J.; Lim, Y.J. Radiosurgical considerations for cavernous sinus hemangioma: Long-term clinical outcomes. Acta Neurochir. 2016, 158, 313-318. [CrossRef] [PubMed]

14. Zhou, L.-F.; Mao, Y.; Chen, L. Diagnosis and surgical treatment of cavernous sinus hemangiomas: An experience of 20 cases. Surg. Neurol. 2003, 60,31-36. [CrossRef]

15. Mori, K.; Handa, H.; Gi, H. Cavernomas in the middle fossa. Surg. Neurol. 1980, 14, 21-31.

16. Rigamonti, D.; Pappas, C.T.E.; Spetzler, R.F.; Johnson, P.C. Extracerebral cavernous angiomas of the middle fossa. Neurosurgery 1990, 27, 306-310. [CrossRef]

17. Nepper-Rasmussen, H.; Bjerre, P.; Jørgensen, K. Endovascular neuroradiologic interventions. Embolization of aneurysms and arteriovenous malformations. Sclerotherapy of cavernous hemangiomas. Ugeskr. Laeger 1993, 155, 3471-3476.

18. Hashimoto, M.; Yokota, A.; Ohta, H.; Urasaki, E. Intratumoral injection of biobond adhesive for removal of cavernous sinus hemangioma. J. Neurosurg. 2000, 93, 1078-1081. [CrossRef]

19. Ohata, K.; El-Naggar, A.; Takami, T.; Morino, M.; El-Adawy, Y.; El-Sheik, K.; Inoue, Y.; Hakuba, A. Efficacy of induced hypotension in the surgical treatment of large cavernous sinus cavernomas. J. Neurosurg. 1999, 90, 702-708. [CrossRef]

20. Li, Z.-H.; Wu, Z.; Zhang, J.T. Surgical Management and Outcomes of Cavernous Sinus Hemangiomas: A Single-Institution Series of 47 Patients. World Neurosurg. 2019, 122, e1181-e1194. [CrossRef]

21. Goel, A.; Muzumdar, D.; Sharma, P. Extradural approach for cavernous hemangioma of the cavernous sinus: Experience with 13 cases. Neurol. Med. Chir. 2003, 43, 112-119. [CrossRef] [PubMed]

22. Suri, A.; Ahmad, F.U.; Mahapatra, A.K. Extradural transcavernous approach to cavernous sinus hemangiomas. Neurosurgery 2007, 60, 483-489. [CrossRef] [PubMed]

23. Goel, A. The extradural approach to lesions involving the cavernous sinus. Br. J. Neurosurg. 1997, 11, 134-138. [CrossRef] [PubMed]

24. Nadkarni, A.G. Cavernous haemangioma in the cavernous sinus. Br. J. Neurosurg. 1995, 9, 77-80. [CrossRef] [PubMed]

(C) 2020 by the authors. Licensee MDPI, Basel, Switzerland. This article is an open access article distributed under the terms and conditions of the Creative Commons Attribution (CC BY) license (http://creativecommons.org/licenses/by/4.0/). 